\title{
An escape of vector matter-wave soliton from a parabolic trap
}

\author{
Yuliy V. Bludov ${ }^{1}$, Monica A. García-Ñustes ${ }^{2}$ \\ ${ }^{1}$ Department of Physics and Center of Physics, University of Minho, PT-4710-057, \\ Braga, Portugal \\ ${ }^{2}$ QuantaLab, University of Minho, PT-4710-057, Braga, Portugal \\ ${ }^{3}$ Instituto de Física - Pontificia Universidad Católica de Valparaíso, Avenida Brasil, \\ Valparaíso, Casilla 2950, Chile. \\ E-mail: bludov@fisica.uminho.pt
}

\begin{abstract}
We show that a vector matter-wave soliton in Bose-Einstein condensate loaded into an optical lattice can escape from a trap formed by a parabolic potential, resembling a Hawking emission. The particle-antiparticle pair is emulated by a low-amplitude bright-bright soliton in two-component Bose-Einstein condensate with effective masses of opposite signs. It is shown that the parabolic potential leads to a spatial separation of BEC components. One component with chemical potential in a semi-infinite gap exerts periodical oscillations, while the other BEC component, with negative effective mass, escapes from the trap. The mechanism of atoms transfer from one BEC component to another by spatially periodic linear coupling term is discussed.

PACS numbers: 03.75.Kk, 03.75.Lm, 67.85.Hj
\end{abstract}

Submitted to: J. Phys. B: At. Mol. Opt. Phys. 


\section{Introduction}

One of the prominent features of the Bose-Einstein condensate (BEC) subjected to the external potential is the possibility to use it as almost a perfect test-bench for reproducing a lot of phenomena from other areas of physics (for review see, e.g. [1]). Particular attention has been paid to the phenomena from condensed matter and cosmology. For instance, an advantage to investigate solid-state phenomena like Bloch oscillations [2, 3], Landau-Zener tunneling [4] and Josephson junction [5, 6] in BEC (instead of proper solid-state structures) lies in the fact that periodic external potential provides an analogue of a perfect crystalline lattice without defects. In the area of cosmology, an interest to both atomic [7- 13] and polaritonic $14-16]$ BECs comes from the possibility to use it as experimentally attainable system of "analogue gravity" [17 a laboratory model for curved-space quantum theory, e.g., black holes and, in particular, Hawking radiation.

Hawking radiation represents an additional emission mechanism of particles from a potential well along with classical escaping by external perturbations [18 20] and quantum tunnelling [20,21]. A simplified view of this process is that quantum fluctuations create a particle-antiparticle pair nearby the black hole edge [22, 23. If one of the pair constituents crosses the event horizon, it never returns, thus giving rise to the emission from the black hole, which in its turn leads to decreasing of black hole energy and mass (for review see, e.g., Ref. [24]). However, Hawking emission from nowadays known astronomic black holes is hard to explore because of its weak predicted intensity. Charged black hole amplification of Hawking radiation can take place owing to the resonance in the cavity formed by inner and outer horizons with subsequent black hole lasing [25, 26]. To realize this idea in BEC a variety of different configurations has been proposed [27 31], and later an experimental realization of lasing from BEC was reported [32]. It is noteworthy that, through the similarity of wave processes, gravity phenomena can be also modelled in others physical systems: electromagnetic wave waveguide [33], slow light in moving medium [34,35 $\$$ or in optical fibers 38,39 , 3 . surface waves on moving water [43, 44], the moving flow with a gradient from subsonic to supersonic flow (so-called acoustic or sonic black hole) 45, 46. Sonic black holes, after being predicted in 1981, was implemented in quantum liquids like liquid He [47].

Another natural advantage of a BEC system is that due to strong two-body interactions BEC matter waves can be considered as localized wavepackets or "solitons", which can propagate at long distances without losing their shape. Solitons possess a particular interest due to their properties typically associated with particle-like states. Thus, solitons may provide interesting insights about particle-like behavior and particleantiparticle interaction. For example, an analogue of Hawking radiation in solitons has

$\ddagger$ Although the possibility to achieve the experimental confirmation of Hawking radiation in this structure is doubtful 36,37

$\S$ Actually, first experimental evidence of Hawking radiation was observed in this system [40, 41, although the nature of observed phenomena is under discussion 42 
been studied within the context of a nonlinear Klein-Gordon equation with applications in Josephson junctions [48]. In addition, binary mixtures of Bose-Einstein condensates can support multicomponent "vector solitons" [49 54]. Loading the BEC in an optical lattice (OL) allows the existence of solitons both in the case of attractive and repulsive interactions [50 53]. In such a case, a two-component BEC can sustain bright-bright and dark-bright stationary solitons [55], mixed-symmetry modes and breathers [54]. Creation of dark-bright soliton in two-component BEC has been already reported in Ref. 56 59]. Meanwhile, the possibility for two-component BEC to be loaded into OL was experimentally demonstrated in Ref. [60,61].

In the present paper, we consider emission of matter wave solitons from a combined parabolic and OL trap [62]. We consider a stationary state of bright-bright solitons in a two-component BEC. Using a periodic sign-varying linear coupling term, we can create a vector soliton, which first and second component chemical potentials are located in the lower and upper edges of the OL spectrum first band. Under such configuration, the first component will have a positive effective mass, meanwhile the second component will have a negative one. In such sense vector soliton serves as an analogue of a particleantiparticle pair. When a parabolic trap is loaded, the first component of vector soliton oscillates periodically inside the trap, while the second one accelerates and escapes from it 63. As an extension of the Hawking radiation concept, we have called our phenomenon as Hawking-like emission, in analogy with Ref. [48].

The paper is organized as follows. In section 2 we expose and describe the model of our problem. In section 3 we represent the stationary state of bright-bright solitons in the two-component BEC and propose a mechanism to create this state. In section 4 we describe the dynamics of two-component soliton in a parabolic trap and the mechanism of a Hawking-like escape of the soliton from the trap. Conclusions and final remarks are presented in section 5 .

\section{The model and preliminary arguments}

To be specific, we consider a spinor BEC composed of two hyperfine states, say of the $\left|F=1, m_{f}=-1\right\rangle$ and $\left|F=2, m_{f}=1\right\rangle$ states of ${ }^{87} \mathrm{Rb}$ atoms 64 .65 confined at different vertical positions by transverse parabolic traps and loaded into an optical lattice of coslike shape. Additionally, a time-dependent external potential $\gamma(x, t)$ (which is aperiodic in general case) is applied to the condensate. At the same time hyperfine states are coupled by a coordinate- and time-dependent coupling field $\beta(x, t)$, which describes transitions between atomic states. Notice that such coupling can be originated by the external magnetic field.

We assume a quasi-one-dimensional cigar-shaped condensate. Then, in the meanfield approximation, the system is described by the Gross-Pitaevskii (GP) equations 66

$$
\begin{aligned}
& i \frac{\partial \psi_{1}}{\partial t}=-\frac{\partial^{2} \psi_{1}}{\partial x^{2}}-V \cos (2 x) \psi_{1}+\gamma(x, t) \psi_{1}+ \\
& \beta(x, t) \psi_{2}+\left(g_{1}\left|\psi_{1}\right|^{2}+g\left|\psi_{2}\right|^{2}\right) \psi_{1},
\end{aligned}
$$




$$
\begin{aligned}
& i \frac{\partial \psi_{2}}{\partial t}=-\frac{\partial^{2} \psi_{2}}{\partial x^{2}}-V \cos (2 x) \psi_{2}+\gamma(x, t) \psi_{2}+ \\
& \beta(x, t) \psi_{1}+\left(g\left|\psi_{1}\right|^{2}+g_{2}\left|\psi_{2}\right|^{2}\right) \psi_{2} .
\end{aligned}
$$

Equations (1) and (2) are written in dimensionless form: OL amplitude $V$, external potential $\gamma(x, t)$ and coupling $\beta(x, t)$ are measured in recoil energy units of $E_{R}=$ $\hbar^{2} \pi^{2} /\left(2 m d^{2}\right)$ (where $m$ is the atomic mass and $d$ is the OL period), while the coordinate $x$ and time $t$ are measured in units of $d / \pi$ and $\hbar / E_{R}$, respectively. At the same time wavefunctions $\psi_{j}(x)$ are measured in the $a_{\perp}^{2} \pi^{2} /\left(4 d^{2}\left|a_{12}\right|\right)$ units, where $a_{\perp}$ is the transverse [in the $(y, z)$-plane] oscillator length, $a_{12}$ is the inter-species s-wave scattering length, thus, $g= \pm 1$ in equations (11) and (2). Respectively, parameters $g_{1}=a_{1} /\left|a_{12}\right|$, $g_{2}=a_{2} /\left|a_{12}\right|$ refer to the intraspecies s-wave scattering lengths in the first and second components, measured in the units $\left|a_{12}\right|$.

In the absence of additional external force and coupling term, $\gamma(x, t) \equiv 0$, $\beta(x, t) \equiv 0$, periodicity of OL gives rise to band-gap structure of both spectrum $E_{n}(q)$ and Bloch functions $\varphi_{n, q}(x)$ in the respective linear problem

$$
E_{n}(q) \varphi_{n, q}(x)=-d^{2} \varphi_{n, q} / d x^{2}-V \cos (2 x) \varphi_{n, q}(x)
$$

(see figure 1(a)). Here $n$ is the band number and $q$ is the Bloch wavenumber in the first Brillouin zone, $q \in[-1,1]$. Meanwhile both the spectrum and the Bloch functions are periodic in the reciprocal space with period 2 (in the chosen units): $E_{n}(q)=E_{n}(q+2)$, $\varphi_{n, q}(x)=\varphi_{n, q+2}(x)$. In further considerations we take into account the first band only $(n=1)$, whose bottom and top correspond to $E_{1}(0)$ and $E_{1}(1)$, respectively, so the index $n$ will be omitted.

\section{Small-amplitude coupled solitons.}

\subsection{Stationary state}

When linear coupling and external force are absent, $\beta(x, t)=\gamma(x, t) \equiv 0$, the coupled nonlinear Schrödinger equations (1) and (2) possess a family of the stationary solutions $\psi_{j}(x, t)=\phi_{j}(x) \exp \left(-i \mu_{j} t\right)(j=1,2)$. Here $\mu_{j}$ are the chemical potentials and $\phi_{j}(x)$ are the soliton profiles of the $j$ th component, governed by the system of the differential equations

$$
\begin{aligned}
& \mu_{1} \phi_{1}=-\frac{d^{2} \phi_{1}}{d x^{2}}-V \cos (2 x) \phi_{1}+\left(g_{1} \phi_{1}^{2}+g \phi_{2}^{2}\right) \phi_{1}, \\
& \mu_{2} \phi_{2}=-\frac{d^{2} \phi_{2}}{d x^{2}}-V \cos (2 x) \phi_{2}+\left(g \phi_{1}^{2}+g_{2} \phi_{2}^{2}\right) \phi_{2} .
\end{aligned}
$$

Without loss of generality, for the solitonic solutions one can consider $\phi_{j}(x)$ to be purely real functions. We also suppose that the chemical potential of the first component soliton $\mu_{1}$ lies in the semiinfinite gap, $\mu_{1}<E(0)$, while chemical potential of second component soliton $\mu_{2}$ is in the first finite gap, $\mu_{2}>E(1)$. When $g_{1}<0, g_{2}>0$, equations (4) and (5) possess a particular approximate solution in the form of low-amplitude coupled soliton 

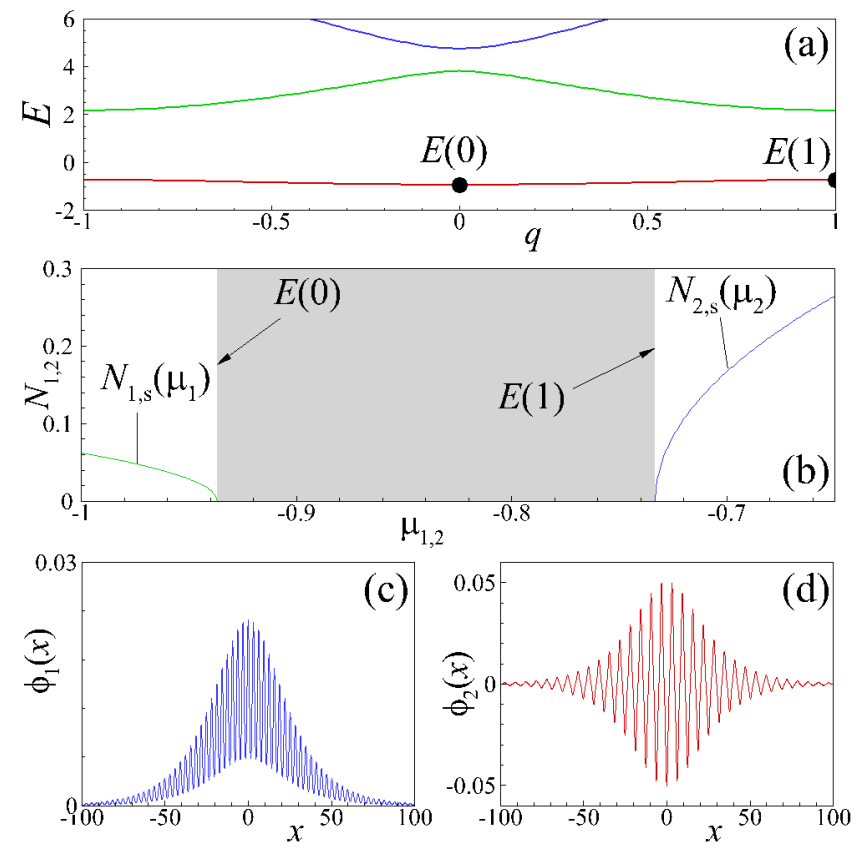

Figure 1. (Color online) (a) Linear band-gap spectrum of OL; (b) First and second component bifurcation diagrams $N_{1}\left(\mu_{1}\right)$ and $N_{2}\left(\mu_{2}\right)$, corresponding to the stationary coupled solution (6), (7); (c,d) Shapes of coupled solitons (6), (7) with chemical potentials $\mu_{1}=-0.938, \mu_{2}=-0.7317$ (soliton norms $N_{1}=0.0085, N_{2}=0.0366$, respectively) - first $\psi_{1}(x, 0)$ and second $\psi_{2}(x, 0)$ components are depicted in panels (d) and (e), respectively. In all panels the parameters of OL and nonlinearities are: $V=3.0, g_{1}=-2, g_{2}=2, g=-1$. The dashed region in the middle of panel (b) are referred to the first band of the OL spectrum.

stationary state. This solution can be obtained [67] by the multiply scale expansion method (see supplementary information for the details) and written as

$$
\begin{aligned}
& \phi_{1}(x) \approx\left(-\frac{1}{M_{0} M_{1} L^{2}} \frac{M_{0} g \chi-M_{1}\left|g_{2}\right| \chi_{1}}{\left|g_{1} g_{2}\right| \chi_{0} \chi_{1}+\chi^{2}}\right)^{1 / 2} \times \\
& \frac{\varphi_{0}(x)}{\cosh (x / L)}, \\
& \phi_{2}(x) \approx\left(-\frac{1}{M_{0} M_{1} L^{2}} \frac{M_{1} g \chi+M_{0}\left|g_{1}\right| \chi_{0}}{\left|g_{1} g_{2}\right| \chi_{0} \chi_{1}+\chi^{2}}\right)^{1 / 2} \times \\
& \frac{\varphi_{1}(x)}{\cosh (x / L)} .
\end{aligned}
$$

Here $M_{q}=\left(d^{2} E(q) / d q^{2}\right)^{-1}$ is the effective mass, $\chi_{q}=\int_{-\pi / 2}^{\pi / 2}\left|\varphi_{q}(x)\right|^{4} d x, \quad \chi=$ $\int_{-\pi / 2}^{\pi / 2}\left|\varphi_{0}(x) \varphi_{1}(x)\right|^{2} d x$

$$
L=\left\{-2 M_{0}\left[\mu_{1}-E(0)\right]\right\}^{-1 / 2}=\left\{-2 M_{1}\left[\mu_{2}-E(1)\right]\right\}^{-1 / 2}
$$

is the soliton width, which is equal in the first and second component of coupled soliton. An example of the coupled soliton shape is represented in figures 1(c) and (d), showing different symmetries of coupled soliton stationary state components (which 
are determined by the symmetries of the respective Bloch functions). In particular, while the first component - the semi-infinite gap soliton - is sign-constant, the second component - the finite gap soliton - is sign-alternating.

Notice, the coupled soliton chemical potentials $\mu_{1,2}$ are not independent variables: the equal width of solitonic components (8) imply the linear character of dependence between chemical potentials, namely

$$
\mu_{2}\left(\mu_{1}\right)=E(1)+\frac{M_{0}}{M_{1}}\left[\mu_{1}-E(0)\right] .
$$

Detuning of the first-component chemical potential from the bottom band edge $E(0)>$ $\mu_{1}$ results into the simultaneous detuning of second-component chemical potential from the top edge of the band $E(1)<\mu_{2}$ (towards the center of the first gap).

The soliton norm $N_{j, s}=\int\left|\phi_{j}\right|^{2} d x(j=1,2)$ in each component of the stationary state, namely

$$
\begin{aligned}
& N_{1, s}\left(\mu_{1}\right)=\frac{2^{3 / 2}}{\pi\left|M_{1}\right|}\left(\frac{E(0)-\mu_{1}}{M_{0}}\right)^{1 / 2} \frac{M_{0} g \chi-M_{1}\left|g_{2}\right| \chi_{1}}{\left|g_{1} g_{2}\right| \chi_{0} \chi_{1}+\chi^{2}}, \\
& N_{2, s}\left(\mu_{2}\right)=\frac{2^{3 / 2}}{\pi M_{0}}\left(\frac{\mu_{2}-E(1)}{\left|M_{1}\right|}\right)^{1 / 2} \frac{M_{1} g \chi+M_{0}\left|g_{1}\right| \chi_{0}}{\left|g_{1} g_{2}\right| \chi_{0} \chi_{1}+\chi^{2}}
\end{aligned}
$$

can be calculated directly from (6), (7), substituting rapidly-oscillating Bloch functions $\varphi_{0,1}(x)$ by their average value on the period $\left\langle\varphi_{0,1}^{2}(x)\right\rangle=\pi^{-1}$. The results are depicted in figure 1(b) in the form of bifurcation diagrams, showing the growth of the soliton norm of both components $N_{1, s}, N_{2, s}$ with an increase of the detuning of chemical potentials from respective band edges.

\subsection{Creation of the stationary coupled state.}

Now a natural question arises: how to create the coupled soliton stationary state? In order to answer it, we introduce the concept of an initial stationary state: the particular solution of Eqs.(1), (2) with all atoms concentrated in the first component,

$$
\begin{aligned}
& \psi_{1, i}(x, t)=\left(\frac{2\left[E(0)-\mu_{1, i}\right]}{\left|g_{1}\right| \chi_{0}}\right)^{1 / 2} \times \\
& \frac{\varphi_{0}(x) \exp \left\{-i \mu_{1, i} t\right\}}{\cosh \left(\sqrt{-2 M_{0}\left(\mu_{1, i}-E(0)\right)} x\right)}, \\
& \psi_{2, i}(x, t)=0 .
\end{aligned}
$$

As the matter of fact, in the initial stationary state, atoms are localized into a bright soliton, whose shape is sign-constant (similar to one, depicted in figure 1(c)). Its soliton norm can be expressed as

$$
\begin{aligned}
& N_{1, i}\left(\mu_{1, i}\right)=\frac{2^{3 / 2}}{\pi\left|g_{1}\right| \chi_{0}}\left(\frac{E(0)-\mu_{1, i}}{M_{0}}\right)^{1 / 2}, \\
& N_{2, i}=0 .
\end{aligned}
$$



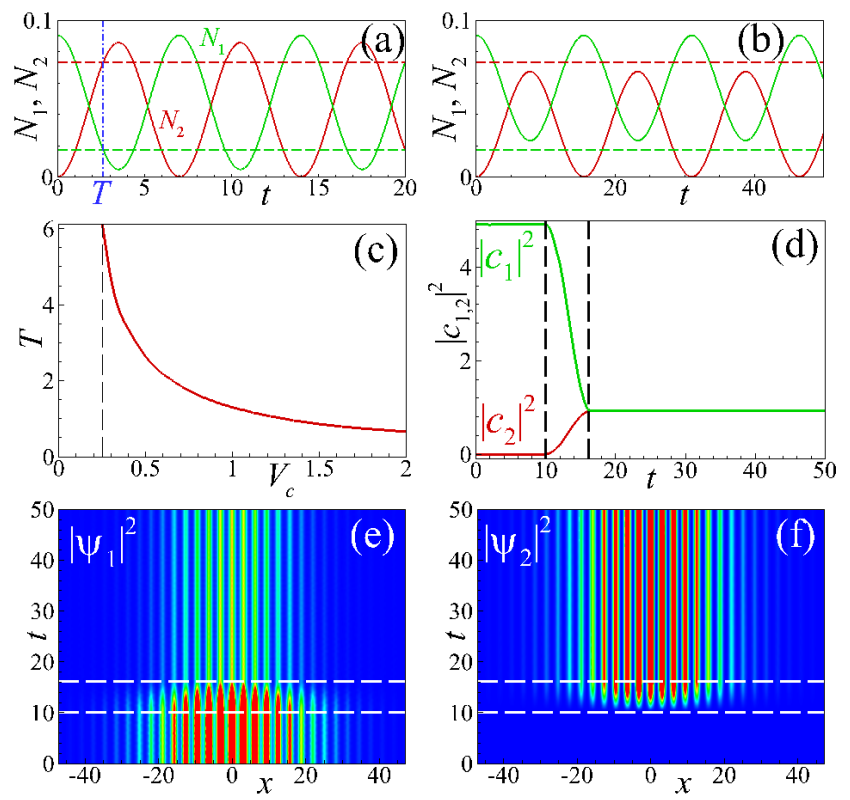

Figure 2. (Color online) (a,b) The temporal dynamics of the number of particles in first (solid green lines) and second (solid red lines) components for the coupling amplitude $V_{c}=0.5$ (a) or $V_{c}=0.2$ (b). The soliton norms in first and second components $N_{1, s}=0.0085, N_{2, s}=0.0366$ are depicted by dashed green and red horizontal lines, respectively; (c) exposure time, necessary to achieve state with soliton norms in first and second components $N_{1, s}, N_{2, s}$, respectively, versus the coupling amplitude $V_{c} ;(\mathrm{d}-\mathrm{f})$ temporal evolution of projections $\left|c_{1,2}(t)\right|^{2}(\mathrm{~d})$ and spatio-temporal evolution of wavefunctions $\left|\psi_{1}(x, t)\right|^{2}$ (e) and $\left|\psi_{2}(x, t)\right|^{2}$ (f) of two-component BEC with linear coupling of the form 18 with $V_{c}=0.25, T_{1}=10, T_{2}=16.12$ (depicted by two dashed lines in b-d). In all panels other parameters are the same as in figure 1 . and initial stationary state is characterized by the first component chemical potential $\mu_{1, i}=-0.94$ and soliton norm $N_{1, i}=N_{1, s}+N_{2, s}$.

Since the system of equations (11) and (2) is conservative, to create a coupled soliton stationary solution with soliton norms $N_{1, s}$ and $N_{2, s}$, one should start from the initial stationary state (12), 13) whose soliton norm is equal to

$$
N_{1, i}\left(\mu_{1, i}\right)=N_{1, s}\left(\mu_{1}\right)+N_{2, s}\left(\mu_{2}\right) .
$$

For the next step, applying nonzero linear coupling between components $\beta(x, t) \neq 0$ for a certain time, one can transfer the atoms from the first component to the second one. Nevertheless, application of a spatially uniform coupling will result in the transferring of the atoms to second component in the same phase, giving rise to similar shapes of solitons in both components. In fact, such resulting soliton will be unstable due to opposite signs of intraspecies nonlinearities $g_{1}$ and $g_{2}$. Notwithstanding, the creation of the coupled soliton stationary state with different symmetries in the first and second components (like in figures 1(c) and 1(d)) is possible if one uses a spatially-periodic linear coupling with the period equal to $2 \pi$,

$$
\beta(x, t)=V_{c} \cos (x) .
$$


Here $V_{c}$ is the amplitude of coupling. Action of the coordinate-dependent coupling (17) gives rise to the periodical oscillations of the number of particles $N_{1}(t)$ and $N_{2}(t)$ in the first and second components of BEC, as is shown in figures 2(a) and (b). If one starts with the initial state (14), (15), a certain amount of particles will be transferred to the second component, and after that will be transferred back, returning to the initial stationary state. By comparing figures 2 (a) and (b) one can see that a growth of the coupling amplitude $V_{c}$ results in the increase of the oscillation amplitude (larger number of particles is transferred to the second component) and the decrease of the oscillation period.

At certain time moment $T$ after the beginning of oscillations the number of particles in each BEC component will be equal to the number of particles $N_{1, s}, N_{2, s}$ (depicted in figures 2(a) and (b) by horizontal dashed lines) of the coupled soliton stationary state, which we want to create. Furthermore, in the text this time will be referred as the exposure time. The dependence of the exposure time $T$, which is necessary to achieve the coupled soliton stationary state shown in figures 1(c) and (d), upon the coupling amplitude $V_{c}$ is represented in figure 2(c). As it is evident, an increasing of coupling amplitude results into the decrease of the exposure time. At the same time, there exists a certain amplitude threshold, designated in figure 2(c) by a vertical dashed line: the desired coupled soliton stationary state can be achieved only for the values of $V_{c}$ above this threshold. For the coupling amplitudes below this threshold the number of particles in first and second BEC components never achieve the values $N_{1, s}, N_{2, s}$ - an example of such situation is shown in figure 2 (b).

It is naturally to presuppose that if the coupling is switched off at the time moment, when the number of particles at first and second BEC component are equal to those of the coupled soliton stationary state (i.e. at time moment equal to the exposure time), then the resulted soliton will keep its shape during long time. This fact is confirmed in figures 2 (d)-(f), where the creation of the coupled soliton stationary state is achieved by switching on the coupling at time moment $T_{1}$ and switching it off after exposure time $T=T_{2}-T_{1}$, namely

$$
\beta(x, t)=\left\{\begin{array}{cc}
0, & t<T_{1} \\
V_{c} \cos (x), & T_{1}<t<T_{2} \\
0, & t>T_{2}
\end{array}\right.
$$

The creation of coupled soliton stationary state is evident from figure 2(d), which represents the projections of the wavefunctions $\psi_{j}(x, t)(j=1,2)$ on the correspondent stationary state $\phi_{j}(x)$ (see equations $(6),(7)$ ), i.e.

$$
c_{j}(t)=\frac{1}{N_{j, s}} \int_{-\infty}^{\infty} \psi_{j}(x, t) \phi_{j}(x) d x .
$$

One observes that after coupling is switched off at time moment $T_{2}$, square modula of these projections are approximately equal to unity during the relatively long integration time. In more details process of the creation of coupled soliton stationary state is depicted in figures 2 (e) and (f). These plots demonstrate both the stability of the initial 


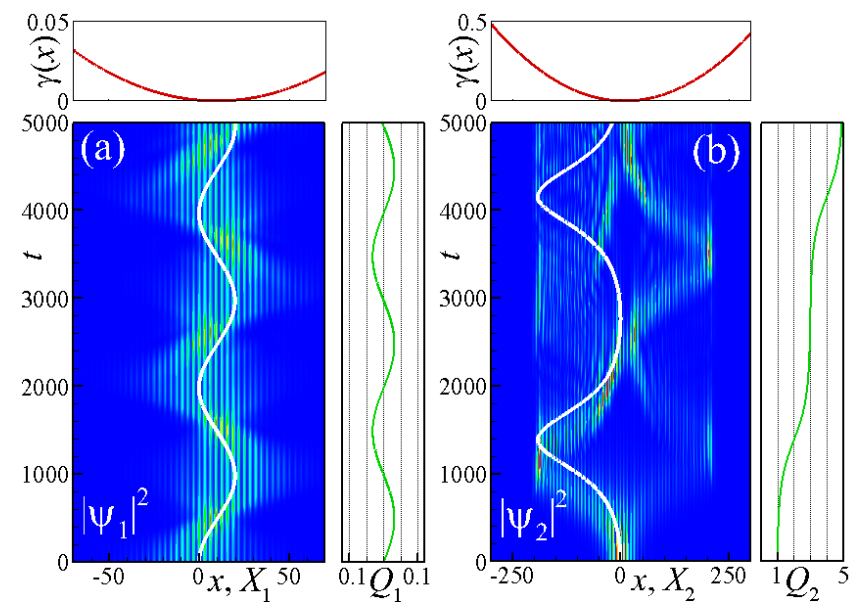

Figure 3. (Color online) Spatio-temporal evolution of particle density $\left|\psi_{1}(x, t)\right|^{2}$ (a) and $\left|\psi_{2}(x, t)\right|^{2}$ (b) (depicted by color maps) in two-component BEC with nonlinearities $g_{1}=-2, g_{2}=2, g=-1$ and loaded into an OL with amplitude $V=3.0$ and parabolic trap (21) with $\nu=5 \cdot 10^{-6}, x_{0}=10$. The initial condition is the stationary gap soliton with $\mu_{1}=-0.938, \mu_{2}=-0.7317$, (depicted in figures 1 1 (c), (d)). Soliton center position in real space $X_{1}(t)$ and $X_{2}(t)$ are depicted by white lines in panels (a) and (b), respectively, while soliton center positions in the reciprocal space $Q_{1}(t)$ and $Q_{2}(t)$ are represented in lateral figures. Values $X_{1}(t), X_{2}(t), Q_{1}(t)$ and $Q_{2}(t)$ were obtained from equations $(25)$ - 28 with parameters $\omega_{0} \approx-0.84, \omega_{1} \approx-0.1$, which correspond to OL with amplitude $V=3.0$. The correspondent shape of the parabolic trap $\gamma(x)$ is presented in two upper figures (notice the different spatial scales of the horizontal axes of panels (a) and (b)).

stationary state at $t<T_{1}$, and the stability of the created coupled soliton stationary state at $t>T_{2}$.

\section{Hawking-like emission of matter from the potential well}

When the additional external potential $\gamma(x, t)$ is applied to the two-component BEC, the dynamics of a localized wavepacket is described by the semiclassical equations [68]

$$
\dot{X}_{j}=\left.\frac{d E}{d q}\right|_{q=Q_{j}}, \quad \dot{Q}_{j}=-\left.\frac{\partial \gamma(x, t)}{\partial x}\right|_{x=X_{j}},
$$

where $X_{j}$ and $Q_{j}$ denote the center of mass of the matter-wave wavepacket's $j$ component in real and reciprocal space, respectively, and the overdot stands for the time derivative. Nevertheless, for the validity of the semiclassical equations 20 the parameters of the BEC should be subject to certain limitations. First, the external potential should be weak, if compared to the OL: its spatial variation on the period of OL should be much smaller than the half-width of the energy band, i.e. $|\gamma(x+\pi, t)-\gamma(x+\pi, t)| \ll|E(1)-E(0)| / 2$. In the opposite case the motion of the wavepacket will be suppressed due to appearance of the Wannier-Stark ladder [68]. This limitation is applicable both in linear and nonlinear cases. Second limitation is specific 
for the nonlinear case only: the soliton width $L$ (see equation (8) ) should be much larger, than the OL period, $L \gg \pi$. Taking into account the inverse dependence between the amplitude of the soliton and its width (see equations (6) and (7)), this requirement can be reformulated as the necessity to use the low-amplitude solitons only. When both these conditions are satisfied, the semiclassical equations (20) describe well [69,70] the dynamics of the soliton in BEC with OL, but only when the modulational instability condition is met. In the modulationally stable regions soliton is destroyed, hence the semiclassical equations are not valid any more.

When the parabolic time-independent potential

$$
\gamma(x, t)=\nu\left(x-x_{0}\right)^{2}
$$

is applied to the two-component soliton, equations (20) can be written as

$$
\begin{aligned}
& X_{j}=x_{0}-\frac{\dot{Q}_{j}}{2 \nu}, \\
& \ddot{Q}_{j}+2 \nu \omega_{1} \pi \sin (\pi Q)=0 .
\end{aligned}
$$

In equations (22), 23) we approximated the band structure $E(q)$ leaving only two leading terms of Fourier expansion, i.e.

$$
E(q)=\omega_{0}+\omega_{1} \cos (\pi q) .
$$

Equation (23) is the differential equation, which describes the oscillation of simple pendulum. Thus, under initial conditions $X_{1}(0)=X_{2}(0)=0, Q_{1}(0)=0, Q_{2}(0)=1$, system of equations (22), (23) possesses an exact solution

$$
\begin{aligned}
& X_{1}(t)=x_{0}\left[1-\operatorname{cn}\left(\frac{\pi \nu x_{0}}{k_{1}} t, k_{1}\right)\right], \\
& Q_{1}(t)=\frac{2}{\pi} \arcsin \left[k_{1} \operatorname{sn}\left(\frac{\pi \nu x_{0}}{k_{1}} t, k_{1}\right)\right], \\
& X_{2}(t)=x_{0}\left[1-\frac{1}{\operatorname{dn}\left(\frac{\pi \sqrt{2 \nu \omega_{1}}}{k_{2}} t, k_{2}\right)}\right], \\
& Q_{2}(t)=\frac{2}{\pi} \operatorname{am}\left(\frac{\pi \sqrt{2 \nu \omega_{1}}}{k_{2}} t, k_{2}\right) .
\end{aligned}
$$

In the above equations

$$
k_{1}=\left(\frac{\nu x_{0}^{2}}{2 \omega_{1}}\right)^{1 / 2}, \quad k_{2}=\left(1+\frac{\nu x_{0}^{2}}{2 \omega_{1}}\right)^{-1 / 2},
$$

are elliptic moduli, $\mathrm{cn}(t, k), \operatorname{dn}(t, k)$ are the Jacobi elliptic functions, $\operatorname{am}(t, k)$ is the Jacobi amplitude.

As it follows from equations (26) and (28), in the reciprocal space, the first component soliton center should exhibit periodical oscillations in the vicinity of $Q_{1}=0$ (see the lateral panel of figure 3(a)). At the same time the soliton center in the second component (the lateral panel of figure $3(\mathrm{~b})$ ) is a increasing function of time\|. In \| with the initial condition $Q_{2}(0)=1$, equation 23 resembles the rotating pendulum 
coordinate space soliton centers of both first and second components exhibit periodic oscillations (see Eqs. 25) and (27)). It is worth noting first component of soliton oscillates in the vicinity of the parabolic trap minimum $x_{0}$, with oscillation period $\tau_{1}=4 K\left(k_{1}\right)\left(2 \pi^{2} \nu \omega_{1}\right)^{-1 / 2}$ and amplitude $\xi_{1}=x_{0}$ (white lines in figure $3(\mathrm{a})$ ), the solitonic second component moves in the region $x<0$ with period $\tau_{2}=2 k_{2} K\left(k_{2}\right)\left(2 \pi^{2} \nu \omega_{1}\right)^{-1 / 2}$ and amplitude $\xi_{2}=\left(\sqrt{\left(2 \omega_{1}+\nu x_{0}^{2}\right) / \nu}-x_{0}\right) / 2$. Again, it is depicted in figure $3(\mathrm{~b})$ by white lines.

These predictions are confirmed by direct numerical integration of equations (1), (2). Figure 3 shows the resulting spatio-temporal evolution of two-component soliton. Nevertheless, while periodical oscillations of solitonic first component are stable, i.e., soliton keeps its shape during long time of evolution, the second component soliton is destroyed after certain time, less than one oscillation period $\tau_{2}$ (see figures 3(a) and (b), respectively). This phenomenon can be explained, if to compare the numerical results with the semiclassical ones. The first component soliton oscillates in the narrow interval in reciprocal space $\sim-0.03 \leq Q_{1} \leq \sim 0.03$ (lateral panel in figure $3(\mathrm{a})$ ), inside which effective mass is always positive, $M_{Q_{1}}>0$. As a result, the modulational instability condition $M_{Q_{1}} g_{1}<0$ is kept at every moment of time, preventing the soliton from the destruction - the semiclassical treatment in the first component is valid in every stage of the process. In contrast, the second component $Q_{2}$ (lateral panel in figure $3(\mathrm{~b})$ ) passes through all values inside the first Brilloin zone, even where the instability condition $M_{Q_{2}} g_{2}<0$ is not met, resulting in the destruction of soliton (notice the above-mentioned non-validity of solutions (25)-28) after the soliton destruction in spite of their formal existence). The quantitative comparison of the numerical results with semiclassical ones shows good correspondence between predicted amplitudes of oscillation in coordinate space and reasonable correspondence between oscillation periods. The discrepancy between periods of oscillation takes place both due to the inexactness of the band approximation (24) and due to inexactness of semiclassical equations 20 in the nonlinear case.

At the initial stage of evolution $t \gtrsim 0$ the positions of the soliton in reciprocal space $Q_{1}$ and $Q_{2}$ increase due to negative $d \gamma(x) / d x$ at $x=0$ (as it follows from equation (20)). In its turn positive $d E(q) / d q$ at $q=0+0$ causes first-component soliton motion to the $x$-positive direction, while negative derivative $d E(q) / d q$ at $q=1+0$ causes that second component moves to the $x$-negative direction, i.e. outwards the parabolic trap center. Similar result for the one-component soliton was demonstrated in Ref. [71]. If the second component of soliton is accelerated during the initial stage, and at certain coordinate $x_{E H}$ the action of external trap is switched off, i.e., $d \gamma(x) / d x=0$, then it will continue its motion with constant velocity, escaping from the parabolic trap. This coordinate $x_{E H}$ can be considered as analogue of event horizon in black hole. In general, the coordinate $x_{E H}$ should be less than the position of second-component soliton center in real space at quarter-period $X_{2}\left(\tau_{2} / 4\right)$. This requirement comes from the necessity to stop action of external force, when the second-component soliton center in the reciprocal space is inside the interval $1<Q_{2}<1.5$ (where effective mass is negative) in order to 
prevent soliton from further destruction.

As an example, this scenario can be realised, when the soliton is placed inside the finite-width parabolic potential (compare with equation (21)

$$
\gamma(x, t)=\left\{\begin{array}{cc}
\nu\left(x-x_{0}\right)^{2}, & \left|x-x_{0}\right|<D / 2, \\
\nu\left(D / 2-x_{0}\right)^{2}, & \left|x-x_{0}\right| \geq D / 2
\end{array},\right.
$$

where $D$ is the width of the potential well. The shape of the potential 29 is depicted in the upper panels of figure 4 . In the frame of the above-mentioned formalism, edges of the finite-width potential (29), $x_{E H 1}=x_{0}-D / 2$ and $x_{E H 2}=x_{0}+D / 2$, can be considered as event horizon analogues. As seen from figures 4(a) and (b), firstcomponent soliton oscillates periodically (see Fig, $4(\mathrm{a})$ ) inside the finite-width potential well (29). Meanwhile, second-component soliton is accelerated during its movement in the negative direction of $x$-axis (see figure $4(\mathrm{~b})$ ), and after crossing the potential-well edge $x_{E H 1}$, depicted by vertical dash-and-dotted line, continues its motion with constant velocity.

Moreover, we can start from the situation when all the BEC atoms are initially concentrated in the first component (like in equations $(12), 130$ ), and then apply, for a finite time, the spatially-periodic linear coupling, which will transfer a portion of atoms to the second component. In other words, we use the same method, as described in section 3, but apply it not to the stationary soliton, but to the oscillating soliton inside the finite-width potential well (see figure $4(\mathrm{c})$ ). In this case atoms, transferred to the second component, will constitute the gap soliton with negative effective mass, which in its turn will escape from the finite-width potential well, as demonstrated in figure $4(\mathrm{~d})$.

\section{Conclusions}

We described a mechanism of stimulated emission of matter waves, in form of bright solitons, from the two-component BEC, loaded into the OL, which is combined with an external parabolic potential. The similarity (in general terms) between the Hawking emission from the black hole and the soliton escape from the parabolic trap is defined by the fact, that we use the bright-bright soliton. Chemical potentials of first and second BEC component lie nearby the opposite edges of the first band of OL spectrum. As a consequence, signs of the effective masses are also opposite, and such type of low-amplitude soliton can be considered as an analogue of a particle-antiparticle pair in Hawking emission. We demonstrated, that this low-amplitude bright-bright twocomponent soliton can be created by partial transferring of atoms from one to another BEC component, using spatially-periodic linear coupling term, whose period equals to the double OL period. Being loaded into the finite-width parabolic trap, one component of such bright-bright soliton with positive effective mass exerts periodic oscillations nearby the trap center, while another component, with negative effective mass, is gradually accelerated and moves in the direction of parabolic trap growth. If soliton with negative effective mass passes the finite-width parabolic trap edge, it escapes from 

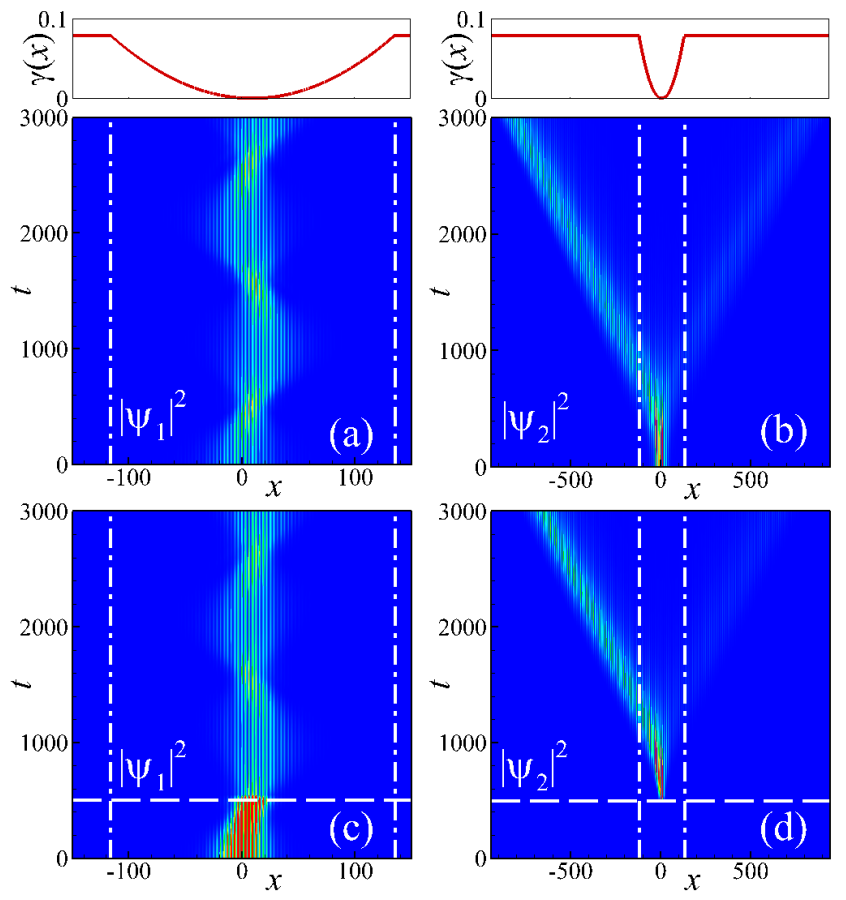

Figure 4. (Color online) Spatio-temporal evolution of particle density $\left|\psi_{1}(x, t)\right|^{2}$ ((a) and (c)) and $\left|\psi_{2}(x, t)\right|^{2}((\mathrm{~b})$ and (d)), obtained from the numerical integration of equations (1), (2) with finite-width parabolic trap (29) and with the linear coupling term $\beta(x, t)=0((\mathrm{a})$ and $(\mathrm{b}))$, or of the form (18) ((c) and (d)) with $V_{c}=0.25, T_{1}=500$, $T_{2}=506.12$ (exposure time is the same as in figure 2). The initial conditions in (a) and (b) correspond to those in figure 3 , and in panels (c) and (d) are the same as in figure 2 In all panels the parameters of OL, finite-width parabolic trap, and nonlinearities are $V=3.0, \nu=5 \cdot 10^{-6}, x_{0}=10, D=80 \pi, g_{1}=-2, g_{2}=2, g=-1$. In all panels edges of the finite-width parabolic trap are depicted by vertical dash-and-dotted lines (the correspondent shape of the finite-width parabolic trap $\gamma(x)$ is presented in two upper figures). In panels (c) and (d) time moments $T_{1}$ and $T_{2}$ are depicted by horizontal dashed lines (indistinguishable in the scale of panels).

the trap and never returns to the initial point. The model proposed above has certain differences with Hawking radiation. Firstly, our analogue of a particle-antiparticle pair, the vector soliton, is created by transferring atoms from first to second components of BEC. In conventional Hawking emission, by contrast, the particle-antiparticle pair is formed from vacuum due to quantum fluctuations. Secondly, in our phenomenon the event horizon is defined somehow artificially as a tunable edge of a potential well.

\section{Acknowledgment}

Y.V.B. acknowledges the support from Portuguese Foundation for Science and Technology (FCT) through Grant No. UID/FIS/04650/2013. M.A.G.-N. thanks for the financial support of FONDECYT project 11130450.

[1] M. Lewenstein, A. Sanpera, V. Ahufinger, B. Damski, A. Sen(De), and U. Sen. Ultracold atomic 
gases in optical lattices: mimicking condensed matter physics and beyond. Advances in Physics, 56(2):243-379, mar 2007.

[2] M. Ben Dahan, E. Peik, J. Reichel, Y. Castin, and C. Salomon. Bloch oscillations of atoms in an optical potential. Phys. Rev. Lett., 76:4508-4511, Jun 1996.

[3] O. Morsch, J. H. Müller, M. Cristiani, D. Ciampini, and E. Arimondo. Bloch oscillations and meanfield effects of Bose-Einstein condensates in 1d optical lattices. Phys. Rev. Lett., 87(14):140402, Sep 2001.

[4] M. Cristiani, O. Morsch, J. H. Müller, D. Ciampini, and E. Arimondo. Experimental properties of Bose-Einstein condensates in one-dimensional optical lattices: Bloch oscillations, Landau-Zener tunneling, and mean-field effects. Phys. Rev. A, 65:063612, Jun 2002.

[5] R. Gati, M. Albiez, J. Fölling, B. Hemmerling, and M. K. Oberthaler. Realization of a single Josephson junction for Bose-Einstein condensates. Appl. Phys. B, 82(2):207-210, 2006.

[6] C. Ryu, P. W. Blackburn, A. A. Blinova, and M. G. Boshier. Experimental realization of Josephson junctions for an atom squid. Phys. Rev. Lett., 111:205301, Nov 2013.

[7] L. J. Garay, J. R. Anglin, J. I. Cirac, and P. Zoller. Sonic analog of gravitational black holes in Bose-Einstein condensates. Phys. Rev. Lett., 85:4643-4647, Nov 2000.

[8] C. Barceló, S. Liberati, and M. Visser. Analogue gravity from Bose-Einstein condensates. Classical Quantum Gravity, 18(6):1137, 2001.

[9] S. Liberaz, M. Visser, and S. Weinfurtner. Analogue quantum gravity phenomenology from a two-component Bose-Einstein condensate. Classical and Quantum Gravity, 23(9):3129-3154, may 2006.

[10] A. Recati, N. Pavloff, and I. Carusotto. Bogoliubov theory of acoustic Hawking radiation in Bose-Einstein condensates. Phys. Rev. A, 80:043603, Oct 2009.

[11] P.-É. Larré, A. Recati, I. Carusotto, and N. Pavloff. Quantum fluctuations around black hole horizons in Bose-Einstein condensates. Phys. Rev. A, 85:013621, Jan 2012.

[12] O. Lahav, A. Itah, A. Blumkin, C. Gordon, S. Rinott, A. Zayats, and J. Steinhauer. Realization of a sonic black hole analog in a Bose-Einstein condensate. Phys. Rev. Lett., 105(24):240401, dec 2010.

[13] D. Boiron, A. Fabbri, P.-É. Larré, N. Pavloff, C. I. Westbrook, and P. Ziń. Quantum signature of analog Hawking Radiation in momentum space. Phys. Rev. Lett., 115:025301, Jul 2015.

[14] D. D. Solnyshkov, H. Flayac, and G. Malpuech. Black holes and wormholes in spinor polariton condensates. Physical Review B, 84(23):233405, dec 2011.

[15] D. Gerace and I. Carusotto. Analog Hawking radiation from an acoustic black hole in a flowing polariton superfluid. Phys. Rev. B, 86:144505, Oct 2012.

[16] H. S. Nguyen, D. Gerace, I. Carusotto, D. Sanvitto, E. Galopin, A. Lemaître, I. Sagnes, J. Bloch, and A. Amo. Acoustic black hole in a stationary hydrodynamic flow of microcavity polaritons. Phys. Rev. Lett., 114:036402, Jan 2015.

[17] C. Barceló, S. Liberati, and M. Visser. Analogue Gravity. Living Rev. Relativ., 14:3, 2011.

[18] P. Hanggi, P. Talkner, and M. Borkovec. Reaction-rate theory: Fifty years after Kramers. Rev. Mod. Phys., 62:251-341, 1990.

[19] S. J. Rooney, A. J. Allen, U. Zulicke, N. P. Proukakis, and A. S. Bradley. Reservoir interactions of a vortex in a trapped three-dimensional Bose-Einstein condensate. Phys. Rev. A, 93:063603, 2016.

[20] S. Potnis, R. Ramos, K. Maeda, L. D. Carr, and A. M. Steinberg. Interaction-assited quantum tunneling of a Bose-Einstein condensate out of a single traping well. Phys. Rev. Lett, 118:060402, 2017.

[21] A. O. Caldeira and A. J. Legget. Quantum tunelling in a dissipative system. Ann. Phys., 149:374456, 1983.

[22] S. W. Hawking. Black hole explosions? Nature (London), 248(5443):30, mar 1974.

[23] S. W. Hawking. Particle creation by black holes. Communications in Mathematical Physics, 43(3):199, 1975. 
[24] D. N. Page. Hawking radiation and black hole thermodynamics. New Journal of Physics, 7(1):203, 2005.

[25] S. Corley and T. Jacobson. Black hole lasers. Phys. Rev. D, 59:124011, May 1999.

[26] U. Leonhardt and G. T. Philbin. Black Hole Lasers Revisited, pages 229-245. Springer Berlin Heidelberg, Berlin, Heidelberg, 2007.

[27] S. Finazzi and R. Parentani. Black hole lasers in Bose-Einstein condensates. New J. Phys., 12(9):095015, 2010.

[28] I. Zapata, M. Albert, R. Parentani, and F. Sols. Resonant hawking radiation in BoseEinstein condensates. New J. Phys., 13(6):063048, 2011.

[29] J. R. M. de Nova, D. Gury-Odelin, F. Sols, and I. Zapata. Birth of a quasi-stationary black hole in an outcoupled Bose-Einstein condensate. New J. Phys., 16(12):123033, 2014.

[30] S. Finazzi, F. Piazza, M. Abad, A. Smerzi, and A. Recati. Instability of the superfluid flow as black-hole lasing effect. Phys. Rev. Lett., 114:245301, Jun 2015.

[31] J. R. M. de Nova, F. Sols, and I. Zapata. Entanglement and violation of classical inequalities in the Hawking radiation of flowing atom condensates. New J. Phys., 17(10):105003, 2015.

[32] J. Steinhauer. Observation of self-amplifying Hawking radiation in an analogue black-hole laser. Nat. Physics, 10(11):864-869, oct 2014.

[33] R. Schützhold and W. G. Unruh. Hawking radiation in an electromagnetic waveguide? Phys. Rev. Lett., 95(3):031301, jul 2005.

[34] U. Leonhardt and P. Piwnicki. Relativistic effects of light in moving media with extremely low group velocity. Phys. Rev. Lett., 84(5):822-825, jan 2000.

[35] U. Leonhardt. A laboratory analogue of the event horizon using slow light in an atomic medium. Nature (London), 415(6870):406-409, 12002.

[36] M. Visser. Comment on relativistic effects of light in moving media with extremely low group velocity. Phys. Rev. Lett., 85(24):5252-5252, dec 2000.

[37] W. G. Unruh and R. Schützhold. On slow light as a black hole analogue. Phys. Rev. D, 68(2):024008, jul 2003.

[38] D. Faccio, S. Cacciatori, V. Gorini, V. G. Sala, A. Averchi, A. Lotti, M. Kolesik, and J. V. Moloney. Analogue gravity and ultrashort laser pulse filamentation. Europhys. Lett., 89(3):34004, feb 2010.

[39] T. G. Philbin, C. Kuklewicz, S. Robertson, S. Hill, F. Konig, and U. Leonhardt. Fiber-optical analog of the event horizon. Science, 319(5868):1367-1370, mar 2008.

[40] F. Belgiorno, S. L. Cacciatori, M. Clerici, V. Gorini, G. Ortenzi, L. Rizzi, E. Rubino, V. G. Sala, and D. Faccio. Hawking radiation from ultrashort laser pulse filaments. Phys. Rev. Lett., 105(20):203901, nov 2010.

[41] E. Rubino, F. Belgiorno, S. L. Cacciatori, M. Clerici, V. Gorini, G. Ortenzi, L. Rizzi, V. G. Sala, M. Kolesik, and D. Faccio. Experimental evidence of analogue Hawking radiation from ultrashort laser pulse filaments. New Journal of Physics, 13(8):085005, aug 2011.

[42] W. G. Unruh and R. Schützhold. Hawking radiation from phase horizons in laser filaments? Phys. Rev. D, 86(6):064006, sep 2012.

[43] G. Rousseaux, C. Mathis, P. Maïssa, T. G. Philbin, and U. Leonhardt. Observation of negativefrequency waves in a water tank: a classical analogue to the Hawking effect? New Journal of Physics, 10(5):053015, may 2008.

[44] S. Weinfurtner, E. W. Tedford, M. C. J. Penrice, W. G. Unruh, and G. A. Lawrence. Measurement of stimulated Hawking emission in an analogue system. Phys. Rev. Lett., 106(2):021302, jan 2011.

[45] W. G. Unruh. Experimental black-hole evaporation? Phys. Rev. Lett., 46(21):1351-1353, may 1981.

[46] Y. Aurégan, P. Fromholz, F. Michel, V. Pagneux, and R. Parentani. Slow sound in a duct, effective transonic flows, and analog black holes. Phys. Rev. D, 92:081503, Oct 2015.

[47] T. A. Jacobson and G. E. Volovik. Event horizons and ergoregions in 3he. Phys. Rev. D, 
58(6):064021, aug 1998.

[48] J. A. González, M. A. García-Ñustes, A. Sánchez, and P. V. E. McClintock. Hawking-like emission in kink-soliton escape from a potential well. New J. Phys., 10(11):113015, nov 2008.

[49] Th. Busch and J. R. Anglin. Dark-bright solitons in inhomogeneous Bose-Einstein condensates. Phys. Rev. Lett., 87:010401, 2001.

[50] V. A. Brazhnyi and V. V. Konotop. Theory of nonlinear matter waves in optical lattices. Mod. Phys. Lett. B, 18(14):627-651, jun 2004.

[51] R. Carretero-González, D. J. Frantzeskakis, and P. G. Kevrekidis. Nonlinear waves in Bose-Einstein condensates: physical relevance and mathematical techniques. Nonlinearity, 21(7):R139-R202, jul 2008.

[52] B. Eiermann, P. Treutlein, Th. Anker, M. Albiez, M. Taglieber, K.-P. Marzlin, and M. Oberthaler. Dispersion management for atomic matter waves. Phys. Rev. Lett., 91(6):060402, aug 2003.

[53] B. Eiermann, Th. Anker, M. Albiez, M. Taglieber, P. Treutlein, K.-P. Marzlin, and M. Oberthaler. Bright Bose-Einstein gap solitons of atoms with repulsive interaction. Phys. Rev. Lett., 92(23):230401, jun 2004.

[54] H. Cruz, V. A. Brazhnyi, V. V. Konotop, G. L. Alfimov, and M. Salerno. Mixed-symmetry localized modes and breathers in binary mixtures of Bose-Einstein condensates in optical lattices. Phys. Rev. A, 76(1):013603, jul 2007.

[55] E. A. Ostrovskaya and Y. S. Kivshar. Localization of two-component Bose-Einstein condensates in optical lattices. Phys. Rev. Lett., 92(18):180405, may 2004.

[56] C. Becker, S. Stellmer, P. Soltan-Panahi, S. Dörscher, M. Baumert, E-V. Richter, J. Kronjäger, K. Bongs, and K. Sengstock. Oscillations and interactions of dark and dark-bright solitons in Bose-Einstein condensates. Nat. Phys., 4(6):496-501, may 2008.

[57] S. Middelkamp, J.J. Chang, C. Hamner, R. Carretero-González, P.G. Kevrekidis, V. Achilleos, D.J. Frantzeskakis, P. Schmelcher, and P. Engels. Dynamics of dark-bright solitons in cigar-shaped Bose-Einstein condensates. Physics Letters A, 375(3):642-646, jan 2011.

[58] C. Hamner, J. J. Chang, P. Engels, and M. A. Hoefer. Generation of dark-bright soliton trains in superfluid-superfluid counterflow. Phys. Rev. Lett., 106(6):065302, feb 2011.

[59] C. Hamner, Y. Zhang, J. J. Chang, C. Zhang, and P. Engels. Phase winding a two-component Bose-Einstein condensate in an elongated trap: Experimental observation of moving magnetic orders and dark-bright solitons. Phys. Rev. Lett., 111(26):264101, dec 2013.

[60] J. M. Higbie, L. E. Sadler, S. Inouye, A. P. Chikkatur, S. R. Leslie, K. L. Moore, V. Savalli, and D. M. Stamper-Kurn. Direct nondestructive imaging of magnetization in a spin-1 Bose-Einstein gas. Phys. Rev. Lett., 95(5):050401, jul 2005.

[61] A. Widera, F. Gerbier, S. Fölling, T. Gericke, O. Mandel, and I. Bloch. Coherent collisional spin dynamics in optical lattices. Phys. Rev. Lett., 95(19):190405, nov 2005.

[62] A. Y. Okulov. 3d-vortex labyrinths in the near field of solid-state microchip laser. Journal of Modern Optics, 55(2):241-259, 2008.

[63] A. Y. Okulov. Superfluid rotation sensor with helical laser trap. Journal of Low Temperature Physics, 171(3):397-407, 2013.

[64] M. R. Matthews, B. P. Anderson, P. C. Haljan, D. S. Hall, M. J. Holland, J. E. Williams, C. E. Wieman, and E. A. Cornell. Watching a superfluid untwist itself: Recurrence of Rabi oscillations in a Bose-Einstein condensate. Phys. Rev. Lett., 83(17):3358-3361, oct 1999.

[65] J. Williams, R. Walser, J. Cooper, E. A. Cornell, and M. Holland. Excitation of a dipole topological state in a strongly coupled two-component Bose-Einstein condensate. Phys. Rev. A, 61(3):033612, feb 2000.

[66] L. P. Pitaevskii and S. Stringari. Bose-Einstein Condensation. International Series of Monographs on Physics. Clarendon Press, 2003.

[67] V. V. Konotop and M. Salerno. Modulational instability in Bose-Einstein condensates in optical lattices. Phys. Rev. A, 65(2):021602, jan 2002.

[68] D. Feng and G. Jin. Introduction to Condensed Matter Physics. Number vol. 1 in Introduction 
to Condensed Matter Physics. World Scientific, 2005.

[69] M. Salerno, V. V. Konotop, and Y. V. Bludov. Long-living Bloch oscillations of matter waves in periodic potentials. Phys. Rev. Lett., 101(3):030405, 2008.

[70] Y. V. Bludov, V. V. Konotop, and M. Salerno. Dynamical localization of gap-solitons by time periodic forces. Europhys. Lett., 87(2):20004, 2009.

[71] H. Sakaguchi and B. A. Malomed. Positive and negative-mass solitons in Bose-Einstein condensates with optical lattices. Mathematics and Computers in Simulation, 69(5-6):492-501, aug 2005 . 